\title{
Conductas de autocuidado de la salud en estudiantes de Enfermería durante la COVID-19
}

\section{Self-care health behaviors in nursing students during COVID-19}

Yenina Angelica Salas Osorio $1, a$

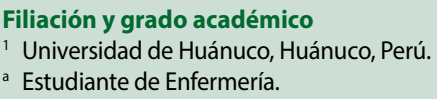

Salas-Osorio YA. Conductas de autocuidado de la salud en estudiantes de Enfermería durante la $\mathrm{CO}$ VID-19. Rev Peru Cienc Salud. 2021; 3(4): 270-7. doi: https://doi.org/10.37711/rpcs.2021.3.4.360

\section{RESUMEN}

Objetivo. Identificar las conductas de autocuidado en la salud física y mental de las estudiantes ingresantes al Programa Académico de Enfermería durante la pandemia de la COVID-19 durante el semestre 2021-1 en la Universidad de Huánuco. Métodos. Fue un estudio cuantitativo, de tipo descriptivo y diseño transversal. La población censal fue 126 estudiantes ingresantes al Programa Académico de Enfermería a quienes se les aplicó una escala de conductas de autocuidado. Se usaron las medidas de frecuencia y proporciones. Resultados. Un 60,9 \% (77) de los estudiantes evidenciaron conductas de autocuidado saludable en la dimensión salud física, frente a un $51,7 \%$ (65) con conducta de autocuidado no saludable en la dimensión salud mental del autocuidado. De modo general, hubo predominio de las conductas de autocuidado saludable [55,8 \% (70)]. Al comparar las características sociodemográficas de la muestra en estudio, según los tipos de autocuidado (saludable/no saludable), se encontró que, tanto entre las mujeres como en el grupo de adolescentes, los estudiantes que se dedican solo al estudio y que viven con sus padres muestran mayor autocuidado saludable. Conclusiones. Existe predominio de las conductas de autocuidado saludable en sus dimensiones: autocuidado de la salud mental y física.

Palabras clave: cuidados de la salud; conductas de salud responsable; COVID-19; prevención del COVID-19; salud mental salud física (fuente: DeCSBIREME).

\section{ABSTRACT}

Objective. To identify self-care behaviors in the physical and mental health of female students entering the Academic Nursing Program during the COVID-19 pandemic in the 2021-1 semester at Universidad de Huánuco. Methods. It was a quantitative, descriptive and cross-sectional study. The sample population consisted of 126 students entering the Academic Nursing Program. A scale of self-care behaviors was applied. Measurements of frequency and proportions were used. Results. $60.9 \%$ (77) of students demonstrated healthy self-care behaviors in the physical health dimension, compared to $51.7 \%$ (65) with unhealthy self-care behaviors in the mental health dimension. In general, there was a predominance of healthy self-care behaviors [55.8\% (70)]. When comparing the sociodemographic characteristics of the sample under study, according to the types of self-care (healthy/unhealthy), it was found that, both among women and in the group of adolescents, students who only study and those who live with their parents show more healthy self-care. Conclusions. There is a predominance of healthy self-care behaviors in the dimensions: mental and physical self-care.

Keywords: health care; responsible health behaviors; COVID-19; COVID-19 prevention; mental health; physical health (source: MeSH NLM). 


\section{INTRODUCCIÓN}

El autocuidado es una compilación de la cultura asociada con el cuidado propio, su familia y de los demás sujetos a su alrededor, que se hace posible partiendo de aspectos como el conocimiento previo, la disponibilidad del tiempo, los recursos económicos, la edad, el género y la inclusión social, así como el uso correcto de los sistemas de salud. Este también implica algunos comportamientos determinados como la asistencia a chequeos médicos, la automedicación, la toma de precaución para evitar accidentes, la observación para identificar alguna irregularidad corporal, la higiene personal, alimentación, hidratación, ejercicios, deporte, recreación ${ }^{(1)}$. El término de autocuidado hace por tanto referencia al grupo de acciones deliberadas que ejecuta la persona en función a sí misma (2).

Los estudiantes evidencian conductas no saludables durante su periodo de formación. La Organización Mundial de la Salud (OMS) ha estimado que, de forma anual, unos 500 millones de jóvenes presentan clamidiasis, gonorrea, sífilis, tricomoniasis y alrededor de 200 millones de mujeres presentan el virus del papiloma humano ${ }^{(3)}$.

En la actualidad no se han hecho estudios sobre el autocuidado en tiempos de la COVID-19; sin embargo, según los estudios presentados previamente, la situación viene incrementándose de forma negativa, ya que la COVID-19 ha afectado tanto a la salud como a la economía y la forma en la que los universitarios se han educado, debido a que, hoy en día, las conductas de autocuidado en los adolescentes son malas ${ }^{(4)}$; por lo que resulta relevante que en este contexto de pandemia se realice un estudio sobre este tema.

Por otra parte, recientes estudios constatan que un $87 \%$ de los adolescentes evidencian obesidad y un $13 \%$ evidencian malos hábitos alimenticios. Lo que permite concluir que los adolescentes no tienen conductas de autocuidado en su alimentación ${ }^{(5)}$, además de pocas conductas de autocuidado, en general ${ }^{\left({ }^{6}\right)}$; así, de acuerdo con la hipótesis se encontró una diferencia en el nivel del "cuidado humanizado" evidenciado en una significancia estadística $(p=0,002)^{(7)}$. En otro estudio, aproximadamente el $81 \%$ de los estudiantes evidenció la práctica de una autoestima regular, el $18 \%$ evidenció mal autocuidado y solo un $1 \%$ evidenció buen autocuidado. En función a las prácticas saludables, el 83,3\% evidenció una alimentación regular, el 65,3\% manejo del estrés y la recreación un $62 \%{ }^{\left({ }^{8}\right)}$.

Otra razón por la que se realizó el estudio fue debido a que la problemática de las enfermedades crónicas presentadas en poblaciones cada vez más jóvenes está en aumento, por lo que se ha vuelto un problema de salud pública que afecta tanto a los mismos estudiantes como a la sociedad en general. Las deficiencias y la falta de interés por el autocuidado que presentan los estudiantes universitarios, tanto a sus labores académicas como a su vida social, hace necesario estudiar esta situación en la Universidad de Huánuco, donde se vienen presentando los mismos problemas ${ }^{(9)}$.

Por ello, este estudio buscó identificar las conductas de autocuidado en la salud física y mental de las estudiantes ingresantes al programa académico de enfermería durante la pandemia de la COVID-19 durante el semestre 2021-1 en la Universidad de , Huánuco, Perú.

\section{MÉTODOS}

\section{Tipo de estudio}

El enfoque del estudio fue cuantitativo. El tipo de estudio fue observacional, descriptivo, y transversal. El estudio se llevó a cabo en la Universidad de Huánuco, Huánuco, Perú, durante los meses de abril y mayo del 2021.

\section{Población y muestra}

Se trabajó con una población censal de 126 estudiantes ingresantes al Programa Académico de Enfermería de la Universidad de Huánuco, en el primer semestre del año 2021, durante la pandemia de la COVID-19. Los criterios de inclusión fueron estudiantes que dieron su consentimiento informado, mientras que el de exclusión fue: estudiantes que se retiraron del semestre o cambiaron de carrera.

\section{Instrumentos de recolección de datos}

Se aplicó la escala de autocuidado de la salud de Ruiz et al. ${ }^{(10,11)}$. Luego fueron incorporadas por la investigadora las modificaciones, al ser una escala de autocuidado relacionada a la pandemia de la COVID-19, para pasar a contener dos dimensiones: salud física y salud mental. A continuación, dentro de cada dimensión se dividen por subdimensiones: física ("alimentación", "actividad física" $y$ "deporte", "higiene" $y$ "bioseguridad"), mental: ("sueño y reposo", "recreación y esparcimiento") y contiene 22 ítems o reactivos. Las respuestas adoptaron un formato tipo Likert con cinco alternativas: nunca, casi nunca, a veces, casi siempre y siempre. Para la medición final fueron consideraron dos categorías de conductas de autocuidado: saludable (124-173 puntos) y no saludables (73-123 puntos).

Para la validación de la escala de autocuidado, esta fue sometida a la revisión de cuatro expertos y tres jueces. Además, se analizó la confiabilidad del instrumento con 
el método del coeficiente de alfa de Cronbach que fue de 0,820 , siendo está por consiguiente muy confiable.

En los instrumentos de recolección de datos también fueron consideradas las preguntas de características generales, como características demográficas, sociales, del estado de salud y de datos informativos.

\section{Procedimientos de la recolección de datos}

La recolección de datos se realizó entre los meses de mayo y junio del 2021; se aplicó un cuestionario de las características generales de la muestra y una escala de conductas de autocuidado de la salud a través de la técnica de encuesta online por la plataforma de Google Forms ${ }^{(12)}$, la cual fue enviada al correo institucional de los participantes con previa aceptación del consentimiento informado virtual. En la primera parte se les detalló el objetivo y la importancia del estudio, luego se hizo el control de calidad de la información recolectada a fin de verificar que todos los datos estén completos, caso contrario fueron eliminados.

\section{Análisis de datos}

Los datos obtenidos fueron procesados en el programa estadístico SPSS, versión 26, en español, procediéndose a utilizar la estadística descriptiva con base en tablas de frecuencias y porcentajes.

\section{Aspectos éticos}

Se consideraron los principios éticos de la Declaración de Helsinki, de este modo, toda la información recolectada se manejó con la reserva y confidencialidad del caso. Se obtuvo el consentimiento informado virtual de los participantes, resumido en la parte superior de la encuesta online; aunque no se llegó a firmar, solo se consideró la opción de si acepta o no participar en el estudio de manera voluntaria.

\section{RESULTADOS}

Al analizar las características demográficas de los estudiantes universitarios en estudio, un mayor porcentaje correspondió al género femenino [65,9\% (83)]. Respecto al grupo etario, el $54 \%$ (68)] eran adolescentes; asimismo, el $40,5(51)$ estudiaba y trabajaba a la vez. Sobre la tenencia de algún seguro de salud, un 56,3 \% (71) no tenía ningún seguro de salud. Respecto a las características de la salud, los cuales muestran que, el $27 \%$ (34) han tenido COVID-19. El 80,2 \% (101) convivía con la familia, y un 94,4\% (119) tenía estado civil soltero. Asimismo, el 73,8 \% (93) usan su laptop para realizar sus clases (ver Tabla 1).
Respecto a las conductas de autocuidado en la dimensión salud física, se observan las preguntas correspondientes al indicador "alimentación", "actividad física" y "deporte, higiene" y "bioseguridad", las cuales muestran que existe una prevalencia sobre las conductas saludables de las estudiantes. Se puede notar que el $56,3 \%$ consumen alimentos con alto valor nutricional, dentro de ellos, pescado y pollo; un $44,4 \%$ consume lácteos y el 49,2\% consume menestras y cereales. En el indicador actividad física y deporte, muestran que no hay una cantidad significante de ellas que realice actividades físicas en casa; sin embargo, hay una tercera parte de estudiantes que aprovechan las tareas domésticas para ejercitarse. En el indicador higiene y bioseguridad, se

Tabla 1. Características sociodemográficas de las estudiantes de Enfermería durante la COVID-19

\begin{tabular}{|c|c|c|}
\hline \multirow{2}{*}{$\begin{array}{l}\text { Características } \\
\text { sociodemográficas }\end{array}$} & \multicolumn{2}{|c|}{$n=126$} \\
\hline & fi & $\%$ \\
\hline \multicolumn{3}{|l|}{ Grupo de edad } \\
\hline Adolescentes & 68 & 54,0 \\
\hline Jóvenes & 36 & 29,0 \\
\hline Adultos & 22 & 17,0 \\
\hline \multicolumn{3}{|l|}{ Género } \\
\hline Masculino & 43 & 34,1 \\
\hline Femenino & 83 & 65,9 \\
\hline \multicolumn{3}{|c|}{ Estudia y trabaja a la vez } \\
\hline No & 75 & 59,5 \\
\hline Sí & 51 & 40,5 \\
\hline \multicolumn{3}{|l|}{ Estado civil } \\
\hline Soltero & 119 & 94,4 \\
\hline Casado & 5 & 4,0 \\
\hline Conviviente & 2 & 1,6 \\
\hline \multicolumn{3}{|l|}{ Tenencia de hijos } \\
\hline Sí & 12 & 9,5 \\
\hline No & 114 & 90,5 \\
\hline \multicolumn{3}{|c|}{ Convivencia con la familia } \\
\hline $\begin{array}{l}\text { Sí } \\
\text { No }\end{array}$ & $\begin{array}{c}101 \\
25\end{array}$ & $\begin{array}{l}80,2 \\
19,8\end{array}$ \\
\hline \multicolumn{3}{|l|}{ Materiales } \\
\hline Laptop & 93 & 73,8 \\
\hline Celular & 21 & 16,7 \\
\hline Computadora & 12 & 9,5 \\
\hline \multicolumn{3}{|c|}{ Problema de salud actual } \\
\hline $\begin{array}{l}\text { Sí } \\
\text { No } \\
\text { Dio positivo al COVID }\end{array}$ & $\begin{array}{c}9 \\
117\end{array}$ & $\begin{array}{l}7,1 \\
92,9\end{array}$ \\
\hline $\begin{array}{l}\text { Sí } \\
\text { No }\end{array}$ & $\begin{array}{l}34 \\
92\end{array}$ & $\begin{array}{l}27,0 \\
73,0\end{array}$ \\
\hline $\begin{array}{l}\text { Tenencia de seguro } \\
\text { Si } \\
\text { No }\end{array}$ & $\begin{array}{l}55 \\
71\end{array}$ & $\begin{array}{l}43,7 \\
56,3\end{array}$ \\
\hline
\end{tabular}


Tabla 2. Descripción del autocuidado de la dimensión salud física de las estudiantes de Enfermería durante la COVID-19

\begin{tabular}{|c|c|c|c|c|c|c|c|c|c|c|}
\hline \multirow{3}{*}{ Autocuidado de la salud física } & \multicolumn{10}{|c|}{$n=126$} \\
\hline & \multicolumn{2}{|c|}{ Nunca } & \multicolumn{2}{|c|}{ Casi nunca } & \multicolumn{2}{|c|}{ A veces } & \multicolumn{2}{|c|}{ Casi siempre } & \multicolumn{2}{|c|}{ Siempre } \\
\hline & fi & $\%$ & $\mathrm{fi}$ & $\%$ & fi & $\%$ & fi & $\%$ & fi & $\%$ \\
\hline \multicolumn{11}{|l|}{ Alimentación } \\
\hline $\begin{array}{l}\text { ¿Consume alimentos con alto valor nutricional en } \\
\text { vitamina C, Ey D? }\end{array}$ & 12 & 9,5 & 12 & 9,5 & 8 & 6,3 & 27 & 21,4 & 67 & 53,2 \\
\hline ¿Consume productos lácteos o derivados? & 34 & 27,0 & 7 & 5,6 & 9 & 7,1 & 20 & 15,9 & 56 & 44,4 \\
\hline ¿Consume pescado y pollo durante la semana? & 2 & 1,6 & 3 & 2,4 & 15 & 11,9 & 35 & 27,8 & 71 & 56,3 \\
\hline ¿Consume menestras y cereal durante la semana? & 9 & 7,1 & 8 & 6,3 & 19 & 15,1 & 28 & 22,2 & 62 & 49,2 \\
\hline ¿Consume mínimo 1 litro de agua al día? & 11 & 8,7 & 20 & 15,9 & 17 & 13,5 & 25 & 19,8 & 53 & 42,1 \\
\hline \multicolumn{11}{|l|}{ Actividad física y deporte } \\
\hline ¿Práctica alguna actividad física recreativa en casa? & 20 & 15,9 & 22 & 17,5 & 18 & 14,3 & 22 & 17,5 & 44 & 34,9 \\
\hline $\begin{array}{l}\text { ¿Aprovecha cualquier tarea doméstica para moverse } \\
\text { o ejercitarse? }\end{array}$ & 16 & 12,7 & 10 & 7,9 & 23 & 18,3 & 28 & 22,2 & 49 & 38,9 \\
\hline \multicolumn{11}{|l|}{ Higiene y bioseguridad } \\
\hline $\begin{array}{l}\text { ¿Te lavas las manos con agua y jabón mínimo } 20 \\
\text { segundos? }\end{array}$ & 7 & 5,6 & 20 & 15,9 & 14 & 11,1 & 20 & 15,9 & 65 & 51,6 \\
\hline ¿Te cepillas los dientes después de cada comida? & 5 & 4,0 & 8 & 6,3 & 6 & 4,8 & 25 & 19,8 & 82 & 65,1 \\
\hline ¿Limpia y desinfecta las superficies de su casa? & 10 & 7,9 & 9 & 7,1 & 13 & 10,3 & 22 & 17,5 & 72 & 57,1 \\
\hline ¿Se desinfectan los calzados al ingresar a casa? & 25 & 19,8 & 18 & 14,3 & 12 & 9,5 & 18 & 14,3 & 53 & 42,1 \\
\hline $\begin{array}{l}\text { ¿Al salir de casa lleva con usted su alcohol para la } \\
\text { desinfección de las manos? }\end{array}$ & 14 & 11,1 & 18 & 14,3 & 19 & 15,1 & 15 & 11,9 & 60 & 47,6 \\
\hline ¿Usa doble mascarilla al salir de casa? & 7 & 5,6 & 20 & 15,9 & 14 & 11,1 & 20 & 15,9 & 65 & 51,6 \\
\hline ¿Usa protector facial al salir de casa? & 29 & 23,0 & 19 & 15,1 & 23 & 18,3 & 11 & 8,7 & 44 & 34,9 \\
\hline ¿Mantiene distancia social cuando sale de casa? & 8 & 6,3 & 9 & 7,1 & 12 & 9,5 & 33 & 26,2 & 64 & 50,8 \\
\hline ¿Evita aglomeraciones y espacios poco ventilados? & 9 & 7,1 & 6 & 4,8 & 13 & 10,3 & 33 & 26,2 & 65 & 51,6 \\
\hline
\end{tabular}

muestra que la mayoría de estudiantes se lavan las vamos por más de 20 segundos, se cepillan los dientes después de cada comida, limpia y desinfecta las superficies de su casa, usa alcohol desinfectante y doble mascarilla; sin embargo, más de la mitad no suele usar mascarilla facial y la mitad de estudiantes no desinfectan sus zapatos al salir o entrar de casa (ver Tabla 2).

Al analizar las conductas de autocuidado en la dimensión salud mental, se observan las preguntas correspondientes al indicador "sueño y reposo" y "recreación y esparcimiento", los cuales muestran que la mayoría de estudiante solo duermen 8 horas diarias e incluso presentan problemas para dormir. Muy pocas incluyen momentos de sueños durante el día. Más de la mitad de estudiantes salen de sus casas a compartir con sus amistades y familia con frecuencia, se sienten satisfechas consigo mismas y son personas con muchos amigos (ver Tabla 3).

Al analizar las dimensiones de las conductas de autocuidado de la salud física de las estudiantes ingresantes a la carrera de Enfermería, se halló que la alimentación es saludable en un $64,3 \%$ de la muestra, seguido de la dimensión higiene y bioseguridad con un $63,5 \%$. Del mismo modo, en el autocuidado de la salud mental se hallaron conductas saludables en la dimensión recreación y esparcimiento con un 65,9% y un 69,1% 
Tabla 3. Descripción del autocuidado de la salud mental de las estudiantes de Enfermería durante la COVID-19

\begin{tabular}{|c|c|c|c|c|c|c|c|c|c|c|}
\hline \multirow{3}{*}{$\begin{array}{l}\text { Autocuidado de la salud mental } \\
\text { Sueño y reposo }\end{array}$} & \multicolumn{10}{|c|}{$n=126$} \\
\hline & \multicolumn{2}{|c|}{ Nunca } & \multicolumn{2}{|c|}{ Casi nunca } & \multicolumn{2}{|c|}{ A veces } & \multicolumn{2}{|c|}{ Casi siempre } & \multicolumn{2}{|c|}{ Siempre } \\
\hline & fi & $\%$ & fi & $\%$ & fi & $\%$ & fi & $\%$ & fi & $\%$ \\
\hline ¿Duerme 8 horas diarias? & 26 & 20,6 & 29 & 23,0 & 10 & 7,9 & 16 & 12,7 & 45 & 35,7 \\
\hline ¿Presenta dificultad o problemas para dormir? & 31 & 24,6 & 19 & 15,1 & 23 & 18,3 & 31 & 24,6 & 22 & 17,5 \\
\hline $\begin{array}{l}\text { ¿Incluye momentos de descanso o siesta de } 10 \text { a } 20 \text { min } \\
\text { en su rutina diaria? }\end{array}$ & 21 & 16,7 & 28 & 22,2 & 27 & 21,4 & 21 & 16,7 & 29 & 23,0 \\
\hline \multicolumn{11}{|l|}{ Recreación y esparcimiento } \\
\hline $\begin{array}{l}\text { ¿Sale de su casa a compartir con sus amistades o } \\
\text { familiares? }\end{array}$ & 17 & 13,5 & 24 & 19,0 & 19 & 15,1 & 27 & 21,4 & 39 & 31,0 \\
\hline ¿Te sientes satisfecho contigo mismo? & 5 & 4,0 & 8 & 6,3 & 13 & 10,3 & 42 & 33,3 & 58 & 46,0 \\
\hline $\begin{array}{l}\text { ¿Tienes amigos a quienes puedes recurrir si necesitas } \\
\text { ayuda? }\end{array}$ & 15 & 11,9 & 6 & 4,8 & 16 & 12,7 & 40 & 31,7 & 49 & 38,9 \\
\hline
\end{tabular}

tuvo conductas no saludables en la dimensión sueño y reposo (ver Tabla 4).

Al analizar las conductas de autocuidado de las estudiantes ingresantes de la carrera de Enfermería de la Universidad de Huánuco, según dimensiones, en una gran proporción de la muestra $(60,9 \%)$ se hallaron conductas de autocuidado saludable en la dimensión de la salud física. Los resultados permiten inferir que la mayoría de estudiantes tienen una buena conducta de alimentación; sin embargo, no todas realizan actividades físicas, frente a un $51,7 \%$ de conducta de autocuidado no saludable en la dimensión salud mental. De modo general, hubo predominio de las conductas de autocuidado saludable 55,8 .

Tabla 4. Dimensiones del autocuidado de la salud física, mental y general de las estudiantes de Enfermería durante la COVID-19

\begin{tabular}{|c|c|c|c|c|}
\hline \multirow{3}{*}{ Dimensiones } & \multicolumn{4}{|c|}{$n=126$} \\
\hline & \multicolumn{2}{|c|}{ No saludable } & \multicolumn{2}{|c|}{ Saludable } \\
\hline & $\mathrm{fi}$ & $\%$ & fi & $\%$ \\
\hline \multicolumn{5}{|l|}{$\begin{array}{l}\text { Conductas de autocuidado de } \\
\text { la salud física }\end{array}$} \\
\hline Alimentación & 45 & 35,7 & 81 & 64,3 \\
\hline Actividad física y deporte & 57 & 45,2 & 69 & 54,7 \\
\hline Higiene y bioseguridad & 46 & 36,5 & 80 & 63,5 \\
\hline \multicolumn{5}{|c|}{$\begin{array}{l}\text { Conductas de autocuidado de la } \\
\text { salud mental }\end{array}$} \\
\hline Sueño y reposo & 87 & 69,1 & 39 & 31,0 \\
\hline Recreación y esparcimiento & 43 & 34,3 & 83 & 65,9 \\
\hline $\begin{array}{l}\text { Conductas de autocuidado } \\
\text { general }\end{array}$ & 56 & 44,2 & 70 & 55,8 \\
\hline
\end{tabular}

Al realizar un análisis cruzado de forma descriptiva de las características sociodemográficas y el nivel de cuidado de la salud de las estudiantes ingresantes de Enfermería, las estudiantes mujeres en el grupo de los adolescentes tienen como única ocupación los estudios y presentan, en mayor medida, una conducta saludable. Las estudiantes que no tienen hijos y que usan la laptop para sus clases presentan también en mayor medida conductas saludables.

\section{DISCUSIÓN}

El presente estudio proporciona una visión general del autocuidado en las estudiantes ingresantes al Programa Académico de Enfermería de la Universidad de Huánuco, basándose en la teoría del Autocuidado de Dorothea Orem ${ }^{(13)}$. De acuerdo con la autora, el autocuidado es un término aprendido por la persona, que persigue un propósito. Asimismo, la teoría de modelo de Virginia Henderson ${ }^{(14)}$. Para la autora, la función de la enfermería es ayudar a las personas enfermas o sanas a poner en práctica actividades que les ayuden al fortalecimiento de su salud, recuperación o una muerte tranquila.

También, el modelo de esencia, cuidado y curación Lydia Hall (15). Esta autora era fiel defensora del agrupamiento de camas en la unidad del servicio de Enfermería terapéutica.

Con respecto a la dimensión cuidado de la salud física, el $60,9 \%$ de estudiantes tienen un nivel alto en el cuidado de la salud física; la mayoría de estudiantes han presentado una conducta saludable de alimentación; sin embargo, no todas las estudiantes 
realizan actividades físicas al igual que sus conductas de higiene y bioseguridad. Con respecto a la dimensión cuidado de la salud mental, el 48,3\% tienen una conducta saludable; sin embargo, la mayoría de estudiantes no realizan reposo; no obstante, sí muestran una conducta de esparcimiento y recreación, lo que en este tiempo resulta siendo una conducta poco responsable. En general, para el cuidado de salud, más de la mitad $(55,8 \%)$ han presentado conductas saludables

Resultados similares al cuidado de la salud mental fueron hallados por Hancco ${ }^{(16)}$, para el autocuidado en estudiantes de Enfermería que asisten a prácticas clínicas en la Universidad Nacional del Altiplano Puno, 2019. Por medio de los resultados se logró conocer que el $81 \%$ de las estudiantes evidenció prácticas de autoestima regular, el $18 \%$ evidenció mal autocuidado, y solo un $1 \%$ evidenció buen autocuidado. Esto permite concluir que, las prácticas de autocuidado en los estudiantes tuvieron una tendencia regular en los estudiantes, no favoreciendo la salud e incidiendo negativamente en la labor de la formación de próximos profesionales. Uno de estos aspectos relacionados al mantenimiento del ejercicio y el deporte en el estilo de vida del sujeto es la motivación, es por ello que la importancia de la misma radica en la práctica de ejercicios físicos, compartir con los amigos y estar a gusto con la práctica. Debido a que el disfrute parece ser la motivación de este tipo de práctica, esta fomenta la unión a las mismas y la promoción de la misma se puede extender a los entornos educativos, donde se pueden realizar distintas actividades que involucran la praxis motriz. Es por ello que en la actualidad se plantea la necesidad de desarrollar tácticas públicas donde se puedan dar respuesta a los adolescentes en función a lo que esperan obtener, intereses, exigencias sociales y juveniles ${ }^{(17)}$.

Los resultados positivos sobre conductas de higiene y bioseguridad tienen mucha semejanza con lo que mencionan Vera et al. ${ }^{(18)}$, en el sentido de que el personal de Enfermería tiene la responsabilidad de prevenir y resguardar la salud de las personas y del lugar donde laboran, dado que se puede dar el riesgo de infecciones y accidentes de trabajo; en tal sentido tiene como responsabilidad el uso de las medidas de bioseguridad. El cumplimiento de estas medidas demanda que el personal y en las demás personas utilicen los conocimientos necesarios que les permitan crear una barrera de protección para prevenir la trasmisión de agentes patógenos entre ellos y otras personas.

Resultados contrarios sobre la alimentación fueron hallados por Ponce en su estudio sobre hábitos de vida no saludables en adolescentes del quinto grado de secundaria de una institución educativa ${ }^{(19)}$. El 27,3\% manifestó que comía 5 o más veces al día, solo un 14,5\% manifestó que realizaba actividades físicas, el 6,6 \% manifestó consumir de 6 a 7 cigarrillos a la semana y el 15,2 \% manifestó que consumía por lo menos más de 5 vasos de cerveza cuando se presentaba la oportunidad. El estudio permitió concluir que los hábitos de vida no saludable guardan una analogía con los problemas de conducta alimentaria.

Asimismo, Quintana y Ruíz ${ }^{(4)}$, en su estudio sobre las prácticas de autocuidado en adolescentes de la Institución Educativa Túpac Amaru, AzapampaHuancayo, 2018, concluyeron que el $24,1 \%$ de los escolares ingerían 8 vasos de agua al día, el 47,6\% manifestó comer las 3 comidas principales del día, $59,2 \%$ manifestó que come continuamente frutas y golosinas y un $31,4 \%$ manifestó que ha tomado cerveza. Lo cual permitió concluir que el conductas de autocuidado en los adolescentes son malas. Chambi ${ }^{(20)}$ presentó el estudio sobre la relación entre factores personales y conductas de autocuidado de la salud en estudiantes de la Escuela Profesional de Enfermería de la Universidad Nacional del Altiplano, Puno-2015. Los resultados de la investigación arrojaron que el $77,5 \%$ tenía conductas de autocuidado de la salud medianamente adecuadas, el 16,3\% conductas inadecuadas y el $6,2 \%$ conductas adecuadas. Lo que permite concluir que la prevalencia en el tipo de conducta de los estudiantes no favorece el autocuidado.

De acuerdo con la salud y los resultados sobre recreación y esparcimiento, Rozo et al. ${ }^{(22)}$ precisan que la recreación se puede definir como un grupo de actividades realizadas por las personas y que se consideran agradables y de satisfacción inmediata; además, estas permiten el desarrollo y fortalecimiento de los valores personales o sociales, así como promover la salud en todos los ámbitos. Este tipo de actividad se desarrolla durante el tiempo libre de las personas, además de fomentar el desarrollo integral de los mismos y permitir el logro de las experiencias significativas por medio del disfrute y el gozo.

La recreación permite incrementar la calidad de vida de la persona, fortalecer su autodesarrollo, potenciar sus habilidades y destrezas, capacidades y actitud física. Asimismo, la recreación es parte de los factores que intervienen en el desarrollo y fortalecimiento de las cualidades y formas de actuar de las personas en función a las normas éticas establecidas por la sociedad. La ocupación del tiempo libre por medio de la recreación 
y el esparcimiento cuenta con tiempo favorable para descansar y producir satisfacción, entretenimiento y placer en las personas.

En síntesis, se evidenciaron conductas de autocuidado saludable en la dimensión de salud física, mientras en la dimensión salud mental se evidenció conducta de autocuidado no saludable. De modo general, hubo un predominio de autocuidado saludable. Al comparar de forma descriptiva las características sociodemográficas de la muestra en estudio, según las conductas de autocuidado (saludable/no saludable), se halló que las mujeres ostentan mayor autocuidado saludable. Asimismo, el grupo de los jóvenes adolescentes tuvo mayor predominio de autocuidado saludable. Las estudiantes ingresantes al Programa Académico de Enfermería que solo se dedican al estudio son las que evidenciaron una mayor proporción de conductas de autocuidado saludables y las estudiantes con condición de solteras sin hijos evidencian una mayor proporción de conductas de autocuidado saludable.

Los resultados obtenidos en la presente investigación constituyen una fuente válida que da sustento a la realización de programas de intervención en los estudiantes ingresantes al Programa Académico de Enfermería durante la pandemia de la COVID-19; frente a ello, es importante mantener a las estudiantes en un nivel de conocimiento adecuado y acertado que les permita orientarse hacia actitudes favorables de prevención y motivación hacia el autocuidado para así modificar las conductas no saludables con el objetivo reconocer los posibles daños o consecuencias y evitar de este modo graves complicaciones a su salud.

\section{REFERENCIAS}

1. Gómez A. Predictores psicológicos del autocuidado en salud. Revista Hacia la Promoción de la Salud. 2017; 22(1): 1-12.

2. Galdemes $S$, Jamet $P$, Bonilla A, Quintero F, Rojas V. Creencias sobre una salud y prácticas de autocuidado en adultos jóvenes: Estudio Biográfico de estilos de vida. Hacia la Promoción de la Salud. 2019; 24(1): 28-43.

3. Infecciones de trasmisión sexual [Internet]. Ginebra: Organización Mundial de la Salud; 2019 [Consultado 2019 Jun 14]. disponible en: http: https://www.who.int/es/ news-room/fact-sheets/detail/sexually-transmitted-infections-(stis)

4. Quintana I, Ruíz K. Práctica de autocuidado en adolescentes de la Institución Educativa Túpac Amaru, Azapampa-Huancayo. 2018 [Internet]. Huancayo: Universidad Peruana Los Andes; 2018. [Consultado 2018 May 23]. Disponible en: https://repositorio.upla.edu.pe/handle/20.500.12848/733

5. Alvarado $M$, Barros $A$. Hábitos alimentarios, estado nutricional de los estudiantes de la Unidad Educativa Manuel Córdova Galarza. 2016 [Internet]. Cuenca:
Universidad de Cuenca; 2017 [Consultado 2017 Jul 20]. Disponible en: http://dspace.ucuenca.edu.ec/bitstream/123456789/27560/1/PROYECTO\%20DE\%20INVESTIGACI\%c3\%93N.pdf

6. Vásquez R. Determinantes de la salud adolescente. Institución Educativa N88016-Chimbote, 2015 [Internet]. Chimbote: Universidad Católica de Los Ángeles de Chimbote; 2017. [Consultado 2017 Abr 7]. Disponible en: http://repositorio.uladech.edu.pe/bitstream/handle/123456789/5546/ADOLESCENTES_DETERMINANTES_VASQUEZ_PENACHI_ROSITA_JACKELYN.pdf?sequence $=1$ \&isAllowed $=\mathrm{y}$

7. Casimiro Z. Calidad de cuidados humanizados que brinda el profesional de enfermería en los servicios de hospitalización de un hospital público de Huánuco-2016 [Tesis de pregrado] Universidad de Huánuco; 2016.

8. Hancco D. Autocuidado en estudiantes de enfermería que asisten a prácticas clínicas, Universidad Nacional del Altiplano, 2018-II [Tesis de pregrado] Puno: Universidad Nacional del Altiplano; 2019.

9. Rivas-Espinoza G, Feliciano-León A, Verde-Flota E, Aguilera-Rivera M, Cruz-Rojas L, Correa-Argueta E, et al. Autopercepción de capacidades de autocuidado para prevención de enfermedades crónicas no transmisibles en estudiantes universitarios. Enfermería Universitaria [Internet]. 2019 [consultado 2019 Jun 14]; 16(1): 5-16. DOI; https://doi.org/10.22201/enero.23958421e.2019.1.57

10. Ruiz-Aquino M, Echevarría Dávila J, Huanca Solis WE. Conductas de autocuidado de la salud en estudiantes universitarios de Ciencias de la Salud. SL [Internet]. 2021 [Consultado 2021 May 3];5(1):261-76. Disponible en: http://revistas.uncp.edu.pe/index.php/socialium/article/view/830

11. Ruiz-Aquino M, Díaz-Lazo A, Ortiz Aguí ML, Enit Ida VC. Conductas de autocuidado frente a la prevención del contagio de la COVID-19 en pobladores peruanos. Rev cuba med gen integr [Internet]. 2020 [Consultado 2021 May 3]; 36(4): Disponible en: http://www.revmgi.sld.cu/ index.php/mgi/article/view/1708

12. Arias J. Métodos de investigación online, herramientas digitales para recolectar datos. Arequipa: Autupublicacion 2020.

13. Naranjo Y, Concepción J, Rodríguez M. La teoría Déficit de autocuidado: Dorothea Elizabeth Orem. Gaceta Médica Espirituana. 2017; 19(3): 1-11.

14. Naranjo Y, Rodríguez M, Concepción J. Reflexiones conceptuales sobre algunas teorías de enfermería y su validez en la práctica cubana. Revista Cubana de Enfermería. 2016; 32(4): 1-13.

15. Raile M, Marriner A. Modelos y teorías en enfermería. 9a ed. Barcelona: ELSEVIER; 2014.

16. Hancco D. Autocuidado en estudiantes de enfermería que asisten a prácticas clínicas, Universidad Nacional del Altiplano, 2018-II [Tesis de pregrado] Puno: Universidad Nacional del Altiplano; 2019.

17. Ahedo R, Macua A. Características de las prácticas de ocio físico-deportivas de los jóvenes españoles. Revista de Psicología del Deporte. 2016; 25(2): 67-72.

18. Vera D, Castellano E, Rodríguez $P$, Mederos T. Efectividad de Guía de buenas prácticas en la bioseguridad hospitalaria. Revista Cubana de Enfermería. 2017; 33(2): 40-51.

19. Ponce J. Hábitos de vida no saludable en adolescentes del quinto grado de secundaria de la Institución Educa- 
tiva Gómez Arias Dávila de Tingo María 2014 [Tesis de pregrado] Tingo María: Universidad de Huánuco; 2018. [Consultado2018 Sep 5]. Disponible en: http://repositorio.udh.edu.pe/bitstream/handle/123456789/876/ T047_42392703_T.pdf?sequence $=1$ \&isAllowed $=y$

20. Chambi A. Relación entre factores personales y conductas de autocuidado de la salud en estudiantes de la Escuela Profesional de Enfermería de la Universidad Nacional del Altiplano Puno 2015 [Tesis de pregrado] Puno: Universidad Nacional del Altiplano; 2017. [Consultado 2017Jul 9]. Disponible en: http://repositorio.unap.edu.
pe/bitstream/handle/UNAP/5626/Chamb\%c3\%ad_Llica_Anali_Katia.pdf?sequence $=1$ \&isAllowed $=y$

21. Valarezo E, Bayas A, Aguilar W, Paredes L, Paucar E, Romero E. Programa de actividades físico-recreativas para desarrollar habilidades motrices en personas con discapacidad intelectual. Revista Cubana de Investigación y Biométrica. 2017; 36(1); 1-13.

22. Rozo-Yugcha A, Aguilar-Chasipanta W, Rodríguez-Torres Á y Jordán-Sánchez J. Efecto de las actividades recreativodeportivas en el estado de ánimo de los adolescentes y jóvenes. Arrancada. 2018; 18(34); 247-257. 